\title{
The assessment of tree row attributes by stratified two-stage sampling
}

Received: 24 February 2005 / Accepted: 9 June 2005 / Published online: 20 September 2005

(C) Springer-Verlag 2005

\begin{abstract}
Tree row inventories are of increasing interest because tree rows mitigate wind erosion and desertification, protect agricultural crops, enhance rural landscape quality, act as bio-corridors, carbon sinks, and a source for bio-energy. The main objective of tree row inventories is to estimate population parameters such as total tree numbers, total tree numbers by species, the mean stem diameter at breast height, the mean tree height and total wood volume. The estimation of these quantities may be straightforwardly carried out whenever aerial images are available in such a way that tree rows can be counted: in these cases, a two-stage cluster sampling may be performed in which the primary units sampled in the first stage are the tree rows in the study area while the secondary units sampled in the second stage are the trees within the selected rows. This paper proposes two sets of two-stage estimators for the interest parameters, based on the Horvitz-Thompson and ratio criteria, together with the corresponding estimators for their sampling variances. The use of stratification is also considered. The proposed procedure was applied to perform a tree row inventory in the Pontina plain (Central Italy): in this case, the tree rows were enumerated by means of ortho-corrected airborne images and stratification was carried out on the basis of the prevailing species and age classes. The inventory results are interesting from a forestry perspective as well as for checking the effectiveness of the procedure.
\end{abstract}

Communicated by Hans Pretzsch

P. Corona $(\bowtie)$

Dipartimento di Scienze dell'Ambiente Forestale e delle sue Risorse, Università della Tuscia, via San Camillo de Lellis s.n.c, 01100 Viterbo, Italy

E-mail: piermaria.corona@unitus.it

Tel: + 39-0761-357425

Fax: +39-0761-357389

L. Fattorini

Dipartimento di Metodi Quantitativi, Università di Siena, Italy
Keywords Linear tree systems - Windbreaks ·

Multiresource forest inventories · Two-stage sampling · Horvitz-Thompson estimators · Ratio estimators · Italy

\section{Introduction}

A current relevant issue in the framework of multipurpose forest inventories concerns surveying trees outside of forests (Kleinn 2000). In particular, surveying tree rows in the landscape, such as windbreaks, hedgerows and other types of linear tree systems, is of increasing interest because tree rows mitigate wind erosion and desertification, protect agricultural crops, enhance rural landscape quality, act as bio-corridors, carbon sinks, and a source for bio-energy (Bellefontaine et al. 2002).

The main objective of tree row inventories is to estimate population parameters such as total tree numbers, total tree numbers by species, mean stem diameter at breast height, mean tree height and total wood volume. Obviously, in any multipurpose inventory the efficient solution should require the use of ad hoc sampling strategies for each of the interest variables. However, in order to save time and resources it is customary to adopt a unique, simple and flexible sampling scheme for all the variables involved (e.g., see Schreuder et al. 1993, p 12). A problem nonetheless arises when adopting the most familiar sampling designs (namely, simple random sampling without replacement and its allied forms) since it is usually prohibitive to obtain the list of trees within the study region. Fortunately, this problem is circumvented when tree rows can be enumerated from aerial photography or similar devices. In these cases, two-stage cluster sampling may be performed in which, in the first stage, a sample of tree rows (the so-called primary units) is selected by simple random sampling without replacement, while, in the second stage, samples of trees (the socalled secondary units) are selected from each of the rows sampled an the first stage, once again by means of simple random sampling without replacement. 